\title{
ESTUDIO DE LOS PROCESOS DE REGENERACIÓN VEGETAL POSTINCENDIO EN PARCELAS EXPERIMENTALES MEDIANTE RADIOMETRÍA DE CAMPO
}

\author{
R. MONTORIO LLOVERÍA(1), F. PÉREZ-CABELLO ${ }^{(1)}$ \\ A. GARCÍA-MARTÍN(1), J. DE LA RIVA FERNÁNDEZ ${ }^{(1)}$
}

(1) Departamento de Geografía y O.T. (Universidad de Zaragoza) C/ Pedro Cerbuna, 12, Zaragoza 50009

Correo electrónico de contacto: montorio@unizar.es

\begin{abstract}
RESUMEN. La vegetación es uno de los componentes de los ecosistemas más afectado por el fuego y su destrucción es, sin duda, el más inmediato y observable de sus efectos. Del mismo modo, los procesos de regeneración vegetal son un claro indicador de la recuperación de los ecosistemas afectados. Estos procesos de regeneración vegetal dependen de la compleja interacción de múltiples factores (bióticos y abióticos) que convergen en diferentes escenarios de intensidad/severidad del fuego. En este contexto, este trabajo tiene como objetivo estudiar los procesos de regeneración vegetal en dos ambientes climáticos contrastados (semiárido y submediterráneo). Para ello, presenta una metodología basada en la quema controlada de parcelas experimentales y en la captura de información espectral con un espectrómetro de campo durante un período de dos años. Los datos recogidos han mostrado importantes diferencias en el comportamiento entre los dos ambientes, diferencias que pueden resumirse en la existencia de rápidos procesos de regeneración vegetal en el ambiente submediterráneo y la ausencia de los mismos en el ambiente semiárido. La escasa influencia mostrada por algunas variables estudiadas como el nivel de vegetación anterior al fuego o el nivel de severidad inmediata permite concluir que la variable climática, en estas comunidades vegetales, es la que realmente condiciona los procesos de regeneración vegetal.
\end{abstract}

ABSTRACT. Vegetation is one of the components of the ecosystems more affected by the fire and its destruction is, indeed, the most immediate and visible of its effects. In the same way, vegetal regeneration processes are a clear sign of the ecosystems recovery. These vegetal regeneration processes depends on the interaction of multiple factors (biotic and abiotic) which take place in different intensity/severity fire scenes. In this context, this work has as objective to study vegetal regeneration processes in two climatically contrasted environments 
(semiarid and subatlantic). It presents a methodology based on the controlled burning of experimental plots and on the capture of spectral information by means of a field spectro-radiometer during a period of two years. Collected data have shown important differences in the behavior of the two experimental stations, differences which can be summarize in the presence of quick vegetal regeneration processes in the subatlantic environment and their absence in the semiarid one. The low influence shown by some analyzed variables such as prefire vegetation level or immediate severity level allows concluding that, in these vegetal communities, the climatic variable is the one which influences vegetal regeneration processes.

Palabras clave: regeneración vegetal, parcelas experimentales, radiometría de campo, ambiente semiárido/submediterráneo.

Key-Words: vegetal regeneration, experimental plots, field radiometry, semiarid-subatlantic environments.

Enviado el 25 de junio de 2007 Aceptado el 20 de octubre de 2007

\section{Introducción}

La destrucción de la cubierta vegetal es quizás el más inmediato y observable de los efectos del fuego sobre los ecosistemas. Del mismo modo, los procesos de regeneración vegetal posteriores al mismo son un claro indicador de la recuperación de los ecosistemas afectados. Los ecosistemas mediterráneos, duramente afectados por los incendios forestales, están constituidos por comunidades vegetales altamente adaptadas a esta distorsión. De este modo, los mecanismos de rebrotamiento y semillado de la mayoría de las especies mediterráneas conducen a un rápido proceso de cubrimiento vegetal, presentando características similares a las comunidades previas (Hanes, 1971; May, 1991).

No obstante, estos procesos de regeneración vegetal presentan una alta variabilidad espacial y temporal, no siendo fácilmente generalizables (Naveh, 1990). La respuesta de las áreas afectadas depende de la compleja interacción entre los diferentes factores bióticos (estrategias de regeneración de las especies afectadas, estructura vegetal, tipo de vegetación...) y abióticos (clima, condiciones meteorológicas posteriores al fuego, topografía, características del suelo...) que tienen lugar en los diferentes escenarios de intensidad/severidad del fuego.

A escala de detalle, en el marco de las parcelas experimentales, el estudio de los procesos de regeneración vegetal ha sido tradicionalmente abordado mediante descripciones cualitativas de la regeneración vegetal: inventarios florísticos, transectos y evaluación visual.

A escala regional, las técnicas de teledetección han demostrado ser una herramienta muy adecuada para estudiar los procesos de regeneración vegetal debido a los cambios generados por el fuego en el comportamiento espectral de la vegetación (Patterson y Yool, 1998; Díaz-Delgado y Pons, 2001). Índices espectrales como el Normalized Differenced Vegetation Index (NDVI) aplicados a imágenes de satélite han sido amplia- 
mente empleados en el seguimiento de los cambios espaciales y temporales de la vegetación y en la estimación de características del fuego (Díaz-Delgado y Pons, 2001; Miller y Yool, 2002; Key y Benson, 2004).

Si bien es cierto que en los últimos años se han hecho importantes avances en la adquisición, procesamiento, análisis e interpretación de datos de alta resolución espectral procedentes de nuevos sensores hiperespectrales y de equipos de adquisición de datos hiperespectrales en el campo (van Wagtendonk et al., 2004; Schaepman et al., 2005), estos estudios a escala de detalle no son tan abundantes.

En este contexto, este trabajo tiene como objetivo estudiar los procesos de regeneración vegetal en dos ambientes climáticos contrastados, mejorando la comprensión de la influencia de las diferentes variables que intervienen en la respuesta de los ecosistemas al fuego. Para ello, se presenta una metodología que, en el marco de las parcelas experimentales y a partir de la información recogida por un espectrómetro de campo sobre un fuego experimental, aplica técnicas de teledetección al estudio de los procesos de regeneración vegetal postincendio.

\section{2. Área de estudio}

El estudio de la regeneración vegetal posterior al fuego se realizó en dos estaciones experimentales. Ambas estaciones se localizan en laderas de orientación sur y con pendiente aproximada de $12^{\circ}$, pero en dos ambientes climáticos muy contrastados: (1) Estación experimental de Peñaflor, localizada en el valle medio del Ebro; (2) Estación experimental de Aisa, localizada en el Prepirineo occidental oscense (Figura 1).

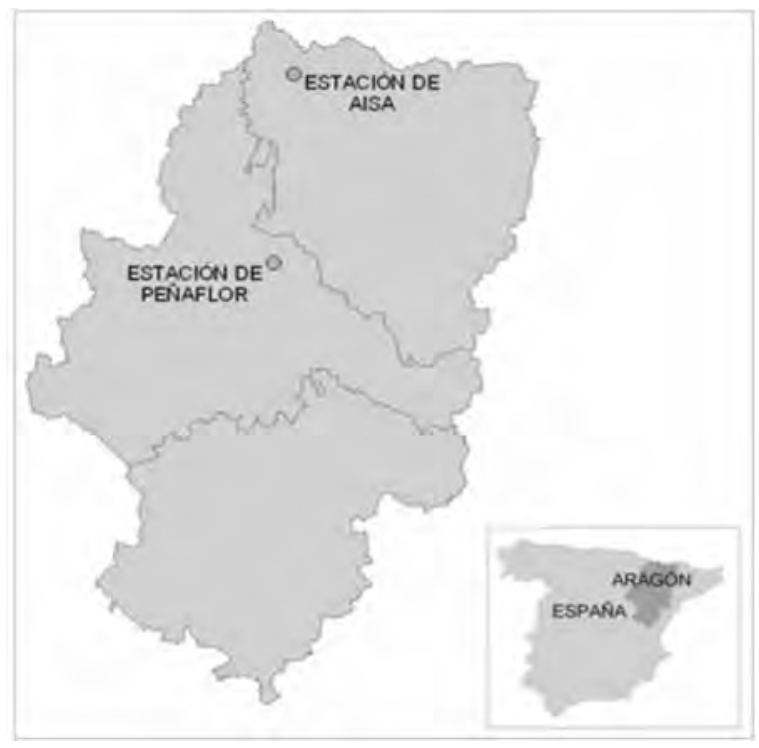

Figura 1. Área de estudio 
La estación experimental de Peñaflor se caracteriza por un clima mediterráneo seco (precipitación media anual de $300 \mathrm{~mm}$. y temperatura media de $15^{\circ} \mathrm{C}$ ). Las parcelas se asientan sobre un Regosol calcáreo desarrollado desde una terraza fluvial cuaternaria y su vegetación es un matorral mediterráneo poco denso dominado por semilladoras -Rosmarinus officinalis- y por herbáceas rebrotadoras -Brachypodium retusum- (Figura 2).

La estación experimental de Aisa presenta un clima de régimen submediterráneo (precipitaciones de régimen equinoccial con media anual de $1.128 \mathrm{~mm}$. y temperatura media anual de $10,4^{\circ} \mathrm{C}$ ). El suelo es un coluvio, compuesto por una matriz limosa de naturaleza calcárea con importantes clastos heterométricos de arenisca. La vegetación del entorno de la estación está dominada por Quercus cerrioides y Pinus sylvestris, acompañados por especies arbustivas como Junniperus communis, Buxus sempervirens, Echinospartum horridum y Genista scorpius. Estas especies arbustivas son las presentes en las parcelas experimentales formando una cubierta vegetal de alta densidad (Figura 2).
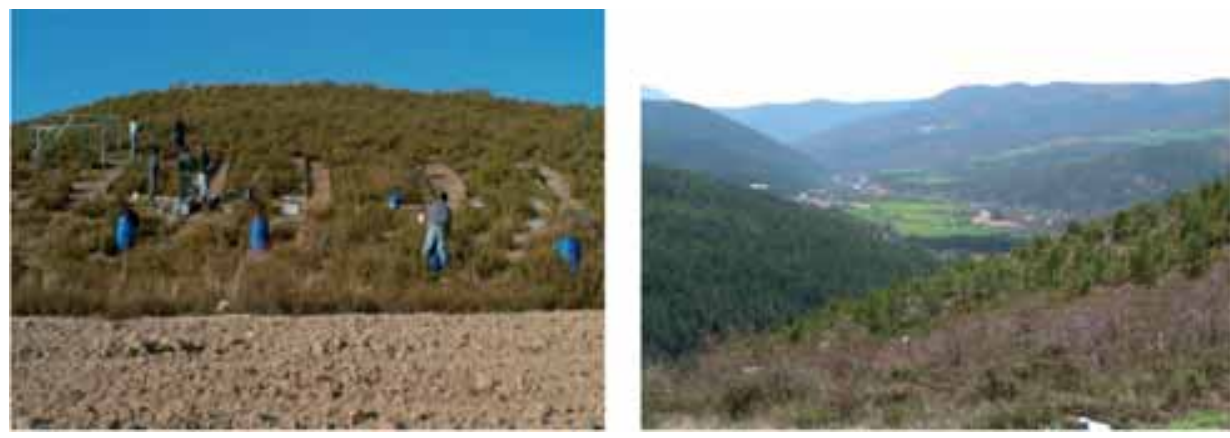

Figura 2. Fotografías de las estaciones experimentales de Peñaflor (izda.) y Aisa (dcha.)

Cada una de estas dos estaciones está constituida por cuatro parcelas experimentales de dimensiones de $3 \times 8 \mathrm{~m}$. En cada estación dos parcelas fueron sometidas a una quema experimental y las otras dos fueron mantenidas como situación control para conocer la evolución natural de los dos ambientes sin la presencia del efecto distorsionador del fuego.

\section{Metodología}

\subsection{Quema controlada de las parcelas experimentales}

El fuego experimental tuvo lugar el 16 de octubre de 2004 en la estación experimental de Peñaflor y el 24 de noviembre de 2004 en la estación experimental de Aisa. En ambos casos el fuego se inició en la parte inferior y se dejó propagar por la superficie de las parcelas de forma natural. Durante el período de fuego activo se registraron algunos parámetros de cara a su posterior caracterización: duración total, velocidad de 
propagación, altura de llama y temperaturas máximas registradas mediante un termómetro sin contacto.

\subsection{Estudio de la regeneración vegetal}

El estudio de la regeneración vegetal postincendio se ha realizado a través del análisis de la evolución de un índice de vegetación, el Normalized Differenced Vegetation Index (NDVI), obtenido a partir de mediciones espectrales realizadas en las parcelas quemadas.

\subsubsection{Medición de la reflectividad espectral}

La obtención de los datos de reflectividad se realizó sobre un conjunto de puntos distribuidos en las parcelas, siguiendo un muestreo regular cada $80 \mathrm{~cm}$. en el eje vertical y $50 \mathrm{~cm}$. en el eje horizontal, que generó un total de 60 puntos por parcela (Figura $3)$. Estos puntos fueron fijados con precisión en las parcelas para asegurar la correcta equivalencia en las diferentes fechas, de forma que los cambios detectados fueran motivados por cambios en el comportamiento de la superficie y no por errores en la georeferenciación de la superficie registrada.
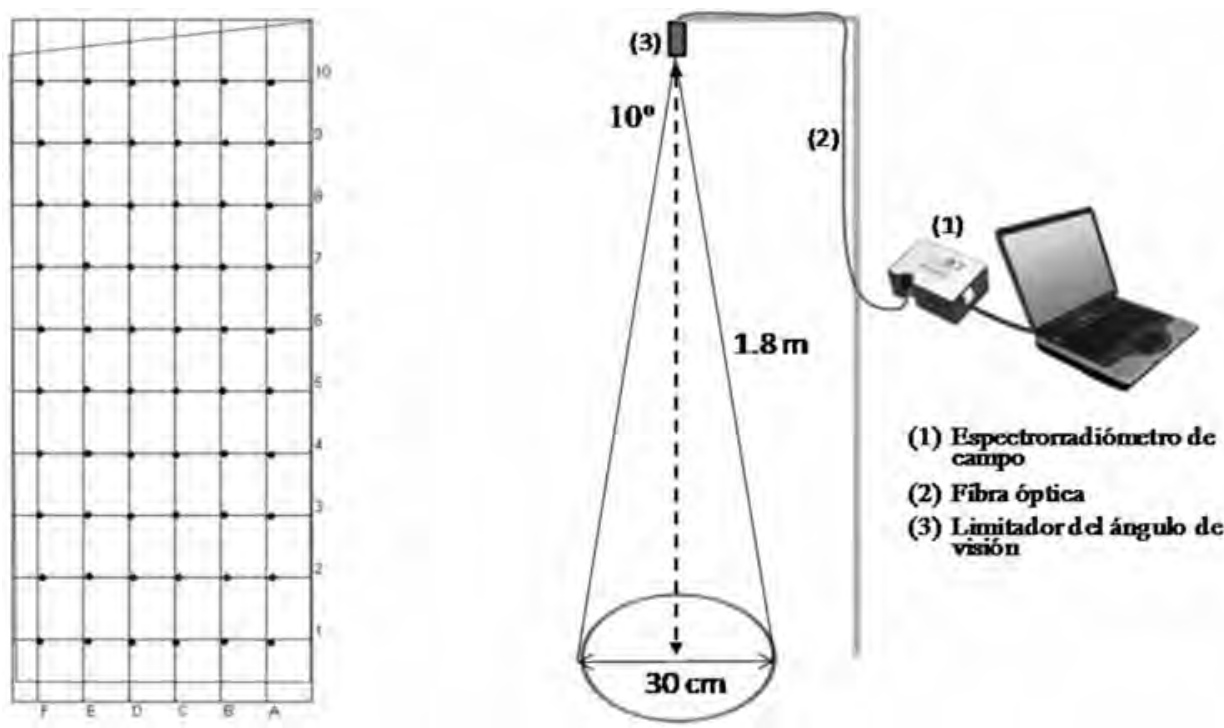

Figura 3. Ejemplo de distribución de los puntos de muestreo en las parcelas experimentales (izda.). Esquema de captura de la reflectividad espectral con un espectrorradiómetro de campo (dcha.)

La frecuencia de mediciones ha variado a lo largo de los dos años considerados en este estudio (Tabla 1). La primera medición se realizó de forma previa al incendio; 
inmediatamente después del mismo se tomó la medida correspondiente a la situación postfuego. Durante el primer año después del fuego las mediciones se realizaron con una frecuencia mensual, al considerarse estos meses los más activos y en los que los procesos se suceden con mayor rapidez e intensidad. Transcurrido un año desde el fuego las mediciones se realizaron con una frecuencia estacional, registrando así las variaciones asociadas a los diferentes momentos del año.

La reflectividad espectral es medida con un espectrorradiómetro de campo (Ocean Optics, USB2000) que registra esta variable en el continuo espectral 400-900 nm, desde el visible (VIS) hasta el infrarrojo próximo (NIR), el sector del espectro más comúnmente aplicado para el estudio de la vegetación, y con una resolución espectral de $0,3 \mathrm{~nm}$.

Tabla 1. Fechas de medición de la reflectividad espectral

\begin{tabular}{|c|c|}
\hline \multicolumn{2}{|c|}{ Peñaflor } \\
\hline Fecha & Meses desde el fuego \\
\hline $15 / 09 / 2004$ & prefuego \\
\hline $16 / 10 / 2004$ & postfuego \\
\hline $21 / 01 / 2005$ & 3 \\
\hline $19 / 02 / 2005$ & 4 \\
\hline $24 / 03 / 2005$ & 5 \\
\hline $05 / 04 / 2005$ & 6 \\
\hline $11 / 06 / 2005$ & 7 \\
\hline $29 / 07 / 2005$ & 8 \\
\hline $26 / 08 / 2005$ & 9 \\
\hline $07 / 12 / 2005$ & 12 \\
\hline $01 / 05 / 2006$ & 18 \\
\hline $11 / 08 / 2006$ & 21 \\
\hline $19 / 01 / 2007$ & 26 \\
\hline & \\
\hline
\end{tabular}

\begin{tabular}{|c|c|}
\hline \multicolumn{2}{|c|}{ Aisa } \\
\hline Fecha & Meses desde el fuego \\
\hline $19 / 10 / 2004$ & prefuego \\
\hline $24 / 11 / 2004$ & postfuego \\
\hline $03 / 01 / 2005$ & 2 \\
\hline $03 / 02 / 2005$ & 3 \\
\hline $12 / 03 / 2005$ & 4 \\
\hline $29 / 04 / 2005$ & 5 \\
\hline $10 / 06 / 2005$ & 7 \\
\hline $07 / 07 / 2005$ & 8 \\
\hline $30 / 08 / 2005$ & 9 \\
\hline $15 / 12 / 2005$ & 12 \\
\hline $11 / 04 / 2006$ & 16 \\
\hline $10 / 08 / 2006$ & 20 \\
\hline $19 / 01 / 2007$ & 25 \\
\hline & \\
\hline
\end{tabular}

Las mediciones se realizaron posicionando el visor del espectrorradiómetro a una altura de $1.8 \mathrm{~m}$. Esta altura, combinada con el ángulo de apertura del visor $\left(10^{\circ}\right)$, generó una superficie de captura de $30 \mathrm{~cm}$. de diámetro para cada uno de los puntos muestreados (Figura 3). Del mismo modo, se procuró la homogeneidad en todas las jornadas de medida: geometría constante entre sensor, panel de referencia y objetivo y similares condiciones atmosféricas, buena iluminación y horario correspondiente al máximo solar (entre las 12 y las 14 h.) para minimizar los efectos debidos a cambios en el ángulo solar. 


\subsubsection{Obtención del índice de vegetación}

El índice de vegetación NDVI está basado en el diferente comportamiento espectral de la vegetación en el continuo de longitudes de onda del espectro electromagnético y, especialmente, en el contraste entre las regiones de rojo $(\mathrm{R})$ e infrarrojo próximo (NIR), las dos regiones que intervienen en su cálculo (Rouse et al., 1974):

$$
N D V I=(N I R-R) /(N I R+R)
$$

En imágenes Landsat, este índice es calculado a partir de la información aportada por las bandas 3 y 4 . Para calcular este índice de vegetación a partir de los datos obtenidos en el campo se decidió obtener la equivalencia del continuo espectral a las bandas del satélite Landsat que, como se ha comentado anteriormente, es uno de los más utilizados en estudios de caracterización de severidad de incendios y de regeneración vegetal (Figura 4). Para llevar a cabo este proceso se tuvo en cuenta la respuesta espectral relativa (Relative Spectral Response, RSR) de las longitudes de onda que componen las bandas del satélite Landsat. A partir de estos valores de RSR se calcularon los correspondientes a las longitudes de onda del radiómetro (debido a que no existía total correspondencia entre las longitudes registradas por ambos sensores) y se calculó la equivalencia a las cuatro primeras bandas de satélite Landsat mediante la siguiente formulación:

$$
\rho B a=\frac{\sum_{n}^{i-m}\left(\rho_{i}^{*} R S R_{i}\right)}{\sum_{n}^{-m} R S R_{i}}
$$

donde $\rho B a=$ reflectividad banda $a ; m$ y $n=$ límites del rango espectral de la banda $a ; \rho i$ $=$ reflectividad en la longitud de onda $i ; R S R i=$ respuesta espectral relativa en la longitud de onda $i$.

Obtenida la equivalencia se procede a calcular el NDVI para cada uno de los puntos muestreados según la formulación anteriormente comentada. Los valores de este índice, que oscilan entre -1 y 1 , pueden ser divididos en intervalos que se corresponden con las diferentes realidades por él representadas. De este modo, una clasificación comúnmente aceptada, que fue empleada en este estudio, establece los siguientes umbrales: (1) valores de NDVI inferiores a 0,2, representativos de suelo desnudo, (2) valores entre 0,2 y 0,4, representativos de áreas mixtas de vegetación y suelo desnudo, (3) valores entre 0,4 y 0,6, representativos de áreas de vegetación de densidad y actividad intermedia y (4) valores superiores a 0,6, representativos de las áreas vegetales de mayor densidad y actividad. Estos umbrales fueron empleados para definir grupos -1 a 4- de puntos de las parcelas quemadas y estudiar la influencia del nivel de NDVI anterior al fuego en la regeneración vegetal postfuego. 


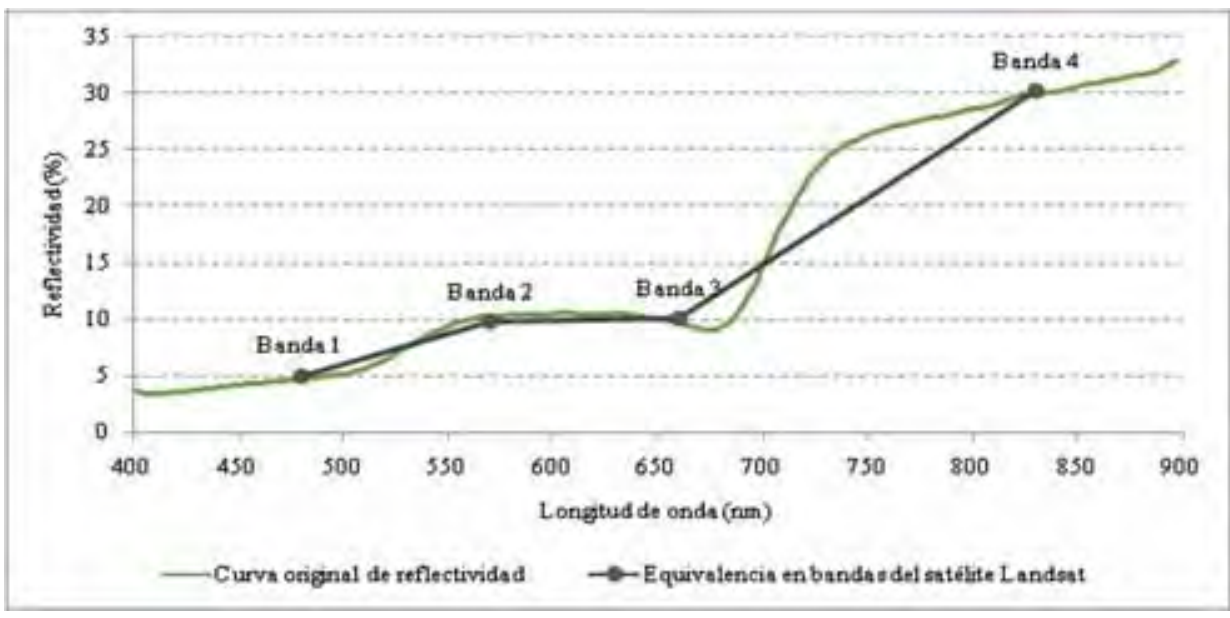

Figura 4. Ejemplo de cálculo de la equivalencia del continuo espectral a las bandas del satélite Landsat en un punto de vegetación activa

Los valores de NDVI promedio de las parcelas quemadas se compararon también con los correspondientes valores promedio de los puntos control seleccionados de vegetación y suelo desnudo en las parcelas no quemadas. Para ello, los puntos de las parcelas fueron subdivididos en dos grupos en función de los umbrales anteriormente comentados: (1) puntos con NDVI inferior a 0,2 para comparar la evolución de los puntos de suelo desnudo; (2) puntos con NDVI superior a 0,4 para comparar la evolución de los puntos de vegetación. Los puntos con NDVI entre 0,2 y 0,4 fueron eliminados de este análisis debido a que su composición mixta podía dificultar la correcta interpretación de los resultados.

Esta comparación permite observar las variaciones de los puntos afectados por el fuego respecto a los puntos control representativos de una situación estable y estimar la evolución del grado de aproximación al punto de control (APC):

$$
\mathrm{APC}=\frac{N D V I \_p q}{N D V I \_p c}
$$

donde $p q$ es el promedio de los puntos quemados y $p c$ es el promedio de los puntos control.

Los valores de APC son iguales a 1 cuando ambos tipos de puntos tienen el mismo comportamiento y son superiores o inferiores a 1 cuando los puntos quemados tienen mayor reflectividad que los puntos control o viceversa. Considerando que los puntos control tienen un comportamiento estable en todo el período y sólo experimentan variaciones fenológicas ligadas al transcurso de las estaciones, el índice APC permite observar tanto las variaciones que sólo tienen lugar en los puntos quemados y que, por tanto, no se explican por dinámica natural de la vegetación, como el proceso de regeneración 
vegetal, considerando que los puntos quemados deben aproximar su comportamiento de forma progresiva al de los puntos control.

\subsubsection{Estimación de la severidad del incendio}

La variable severidad del incendio ha sido estimada en este estudio a partir de la información radiométrica, caracterizando los puntos en función de la pérdida de NDVI. El índice empleado ha sido el dNDVI, basado en la diferencia de valor de NDVI entre los momentos pre- y postfuego.

$$
d N D V I=(\text { NDVIprefuego }- \text { NDVI postfuego }) * 1000
$$

Los valores del índice dNDVI pueden agruparse en intervalos de severidad (Tabla 2). En este estudio se han empleado los umbrales establecidos por Key y Benson (2004) para el índice de severidad dNBR, obtenido con la misma formulación que el dNDVI pero con la información de la banda 7 del satélite Landsat en lugar de la banda 3.

El cálculo del índice dNDVI entre los momentos pre-y postfuego permitió estimar la severidad inicial del fuego sobre las parcelas. Del mismo modo, el cálculo de este índice entre el momento temporal prefuego y la sucesión de fechas posteriores permitió estudiar la evolución de la severidad en el tiempo.

$$
d N D \text { VImesx }=(\text { NDVIprefuego }- \text { NDVImesx }) * 1000
$$

Tabla 2. Intervalos de severidad a partir del índice dNDVI

\begin{tabular}{|c|c|}
\hline Valores de dNDVI & Intervalo de severidad \\
\hline-250 a -101 & Revitalización \\
\hline-100 a 99 & Nula \\
\hline 100 a 269 & Baja \\
\hline 270 a 439 & Media-baja \\
\hline 440 a 659 & Media-alta \\
\hline 660 a 1300 & Alta \\
\hline
\end{tabular}

\section{Resultados}

\subsection{Características del fuego experimental}

La diferente distribución de la vegetación en las parcelas de ambas estaciones experimentales provocó diferencias en el comportamiento del fuego. La discontinuidad del combustible en las parcelas de Peñaflor dificultó la propagación del fuego, que fue 
manualmente reactivado en diferentes ocasiones, y tuvo una duración total de 20 minutos. Esta baja densidad generó también una baja intensidad del incendio, alcanzando alturas de llama de $1 \mathrm{~m}$. aproximadamente y temperaturas máximas registradas en el rango $400-600^{\circ} \mathrm{C}$ (Figuras 5 y 6 ).
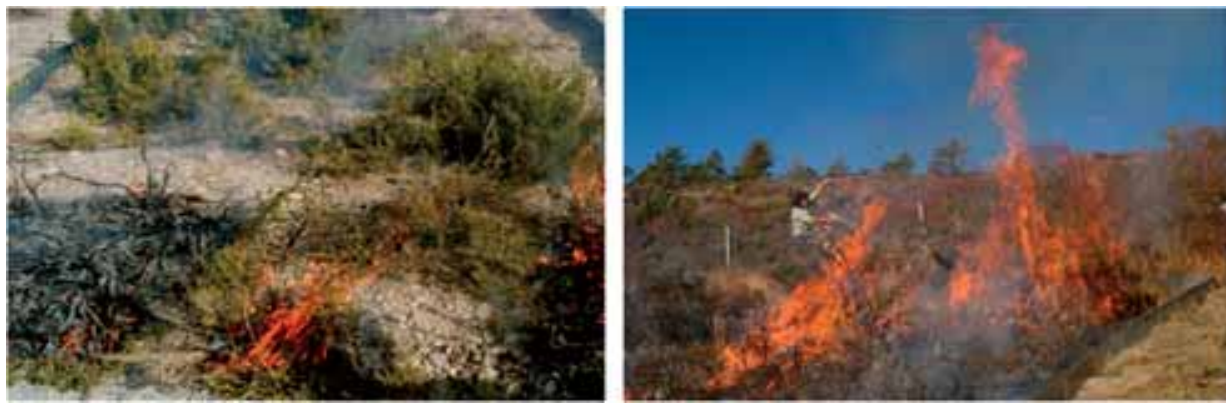

Figura 5. Fotografías del fuego controlado en las parcelas: Peñaflor (izda.), Aisa (dcha.)

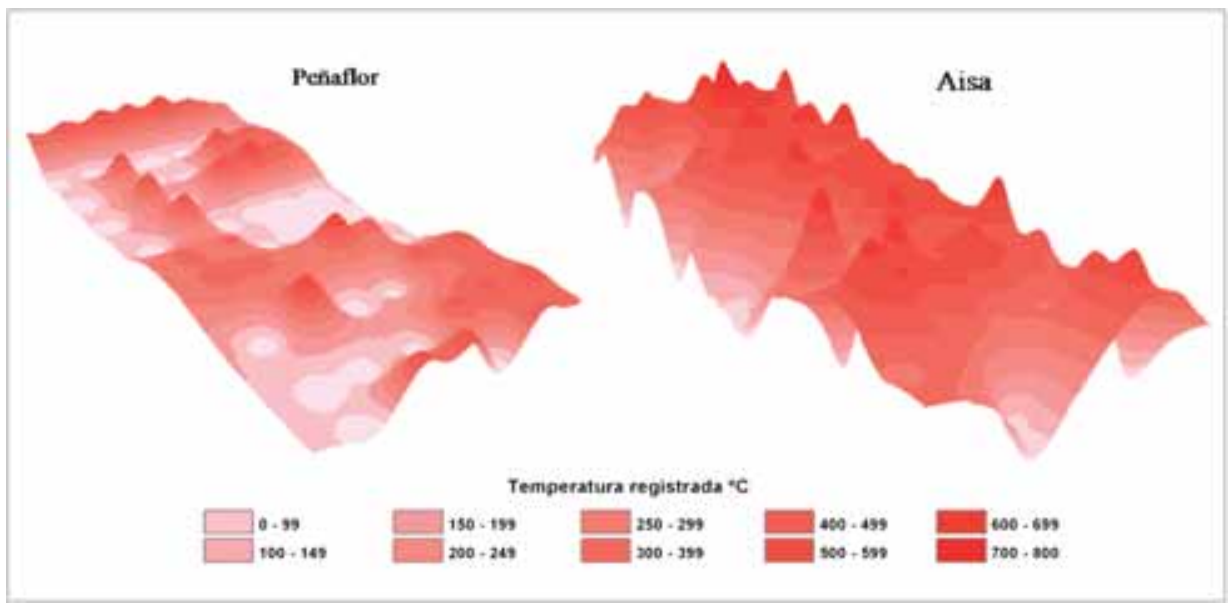

Figura 6. Distribución espacial de las temperaturas máximas del fuego controlado

La continuidad y densidad del combustible en las parcelas de Aisa favoreció la propagación natural del fuego y una elevada intensidad del mismo. El fuego afectó toda la superficie de la parcela en un tiempo de 5 minutos, con alturas de llama entre 2-3 m. y temperaturas máximas registradas en el rango $700^{\circ} \mathrm{C}$ (Figuras 5 y 6). 


\subsection{Estación experimental de Peñaflor}

\subsubsection{Análisis de la regeneración vegetal postincendio}

Las dos parcelas de la estación experimental de Peñaflor, observados sus valores promedio individuales, muestran un comportamiento idéntico en todo el período estudiado (Figura 7). Este hecho permite estudiar sus valores promedio conjuntos como representativos de la evolución del NDVI después del fuego en un ambiente semiárido.

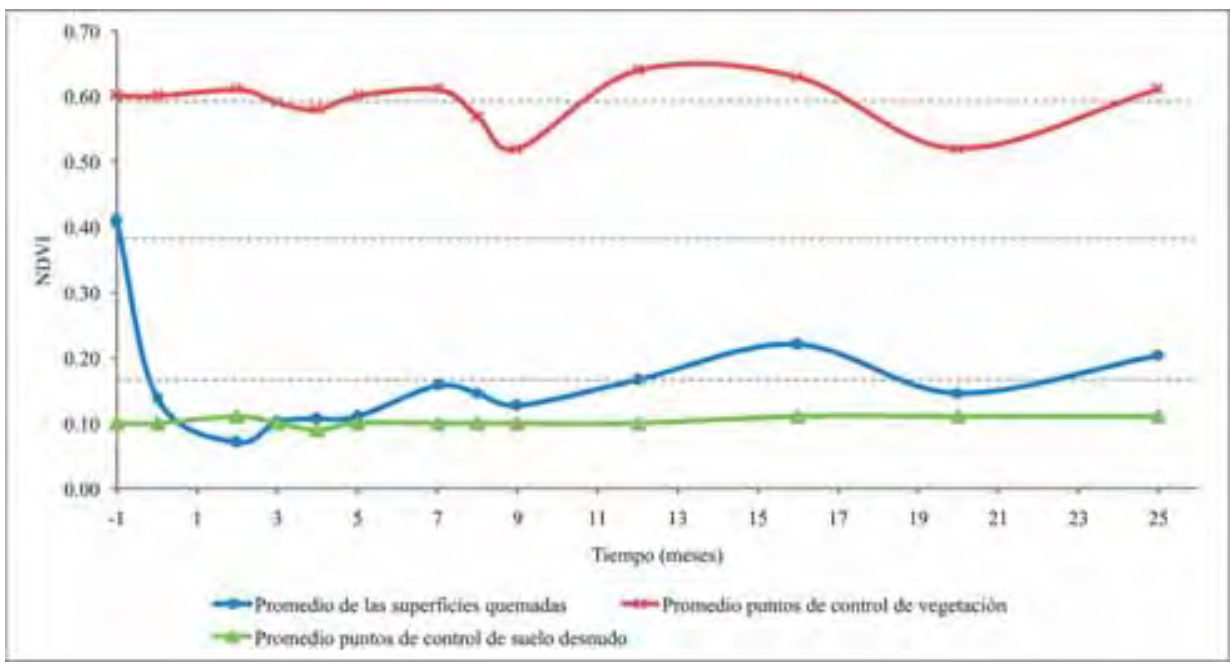

Figura 7. Evolución del NDVI en la estación experimental de Peñaflor

Las parcelas de la estación experimental de Peñaflor parten de una situación previa al fuego con un valor de NDVI de 0,4 (Figura 8). Según este valor, y teniendo en cuenta la anteriormente comentada clasificación de valores de NDVI, pueden considerarse como parcelas con cubierta vegetal de escasa densidad, descripción que corresponde a la realidad observable en el campo (Figura 9). El fuego controlado provocó una desaparición total de la cubierta vegetal y supuso un descenso del NDVI a un valor de 0,15 , situación que puede calificarse como de suelo desnudo (Figura 9). Aunque visualmente pueden observarse restos vegetales en el momento temporal postfuego en algunas localizaciones, ésta es muy escasa y se encuentra seriamente dañada. La información radiométrica en estos puntos no muestra el contraste característico de la vegetación activa entre las regiones rojo e infrarrojo próximo. 


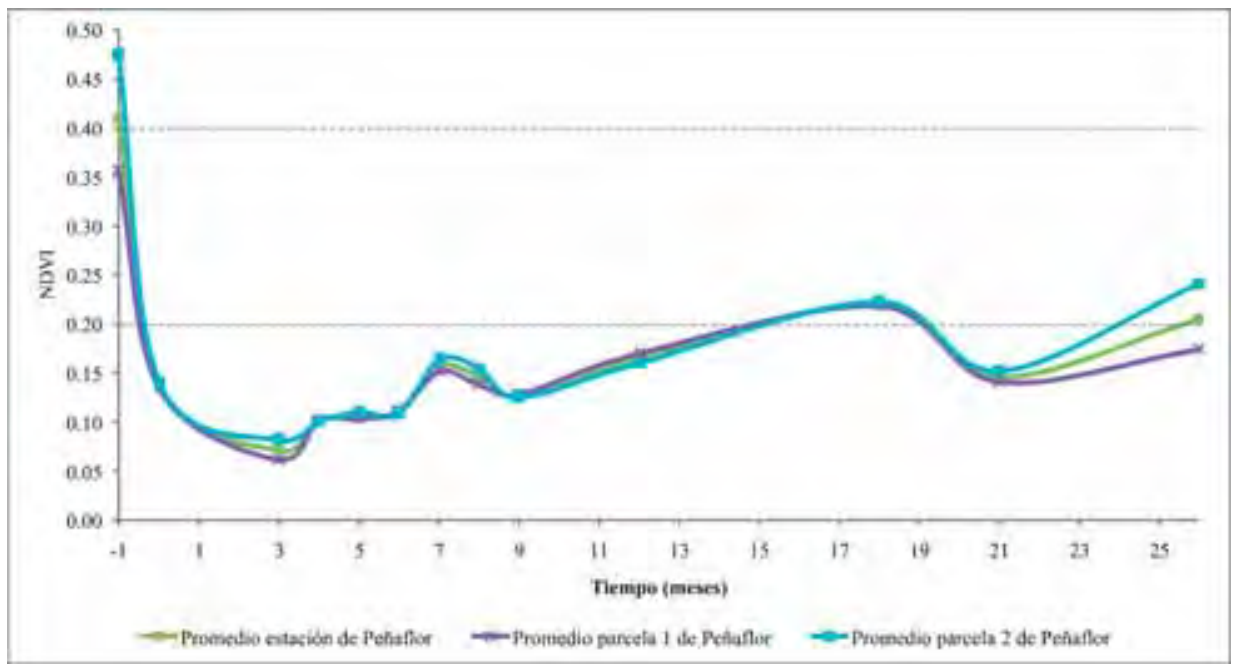

Figura 8. Evolución del NDVI en la superficie quemada de la estación de Peñaflor
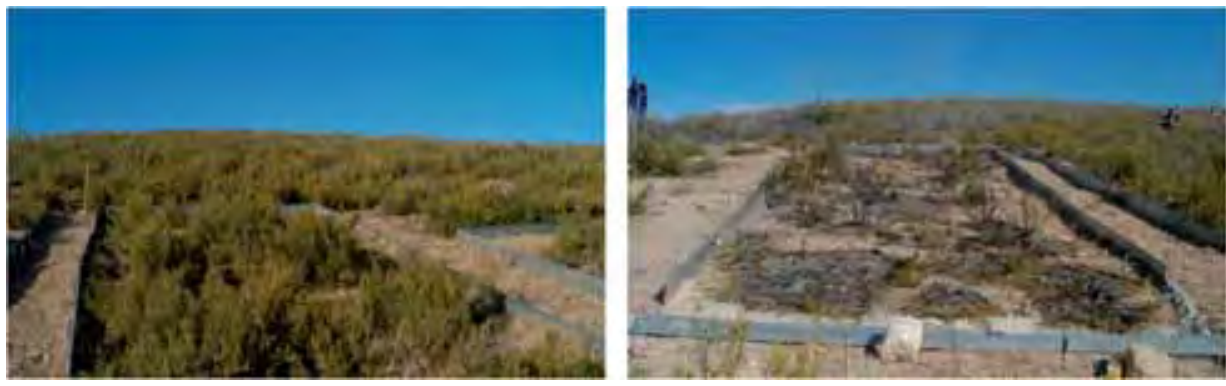

Figura 9. Fotografías de la estación de Peñaflor en los momentos prefuego (izda.) y postfuego (dcha.)

Un mes después del fuego el NDVI continúa descendiendo hasta valores por debajo de 0,1 . Este descenso es explicado por la mortalidad de algunos individuos que sobrevivieron al incendio.

Desde este momento temporal el NDVI evoluciona con una tendencia ascendente hasta el final del período de estudio. En esta tendencia general ascendente se observan ciclos de ascenso/descenso del NDVI que corresponden a las variaciones fenológicas de la vegetación en las diferentes estaciones del año, hecho demostrado por la perfecta correspondencia de estos ciclos con los experimentados por los puntos de control (Figura 8).

Esta tendencia ascendente supone un incremento del NDVI desde valores de 0,08 un mes después del fuego a valores de 0,2 al final del período. El valor promedio de las 
parcelas se sigue manteniendo dos años después del fuego en el intervalo que puede clasificarse como suelo desnudo, indicando una ausencia total de procesos de regeneración vegetal.

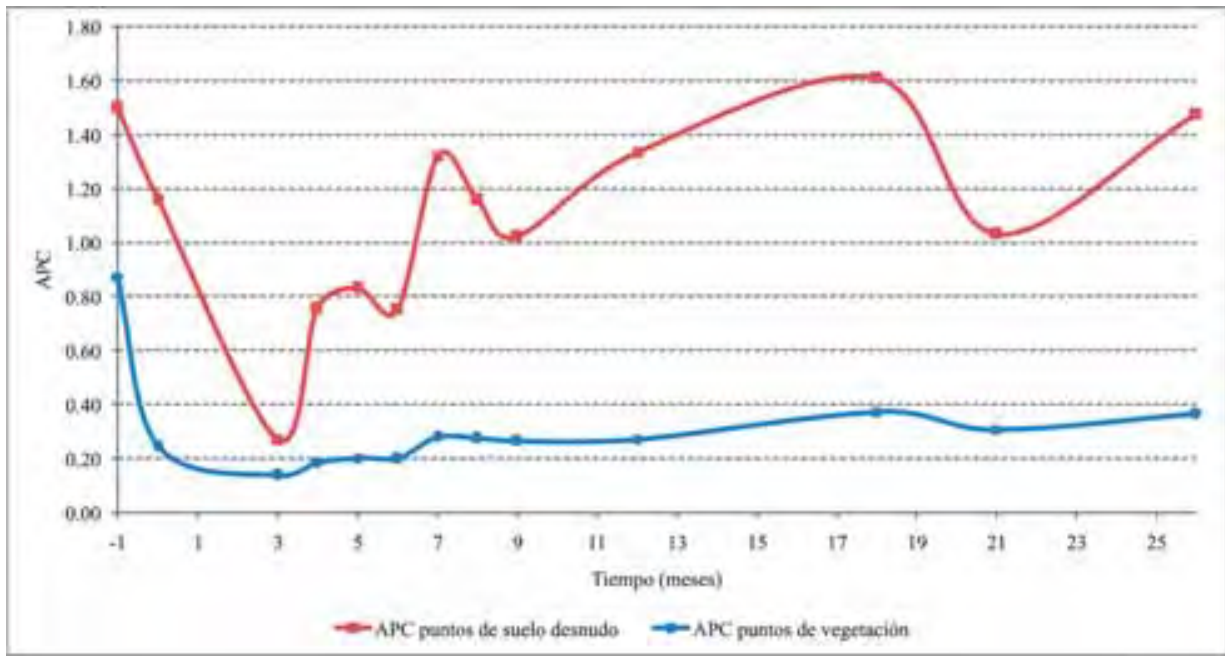

Figura 10. Evolución comparativa del APC en las parcelas de Peñaflor

4.2.2. Evolución comparativa respecto a la situación control

\section{Evolución de los puntos de suelo desnudo}

La evolución de los puntos control de suelo desnudo refleja una total estabilidad en el período estudiado, con valores constantes de 0,1 (Figura 8). La comparación de los puntos de suelo desnudo de las parcelas con este suelo control muestra claramente el diferente comportamiento de estos dos tipos de puntos (Figura 10), diferencia que existe incluso en el momento prefuego donde el valor de APC es de 1.5. Así pues, es este valor el que significaría la situación de estabilidad respecto al momento prefuego.

La variación de los puntos de suelo desnudo de las parcelas quemadas es muy acusada experimentando fuertes ascensos y descensos hasta alcanzar en el momento final estudiado un valor muy similar al registrado en el prefuego. Estas fuertes variaciones se explican porque los puntos de suelo desnudo de las parcelas quemadas, a pesar de no haber sufrido los efectos directos del fuego, sí han sufrido los efectos indirectos derivados de la desaparición de la vegetación circundante. El cubrimiento por las cenizas generadas en la combustión y posteriormente dispersadas por las parcelas modificó los valores de reflectividad en el momento inmediatamente posterior al fuego. En los meses sucesivos, la pérdida de la vegetación supuso una mayor exposi- 
ción a los agentes erosivos (precipitación y viento) y, en consecuencia, un incremento en los valores de pérdida de suelo (desaparición del material fino e incremento de la pedregosidad). El valor de APC registrado en la última fecha estudiada (1.47) no indica correspondencia con la situación control pero sí estabilidad respecto a la situación inicial después de las fuertes variaciones registradas durante los dos años posteriores al fuego.

\section{Evolución de los puntos de vegetación}

Los puntos control de vegetación se mantienen en todo el período de estudio en el intervalo de NDVI entre 0,5 y 0,7 . Estos puntos experimentan ciclos de ascenso/descenso de NDVI ligados a las variaciones fenológicas de la vegetación (Figura 8).

Los puntos de vegetación de las parcelas quemadas no muestran una correspondencia perfecta con los puntos control, el valor registrado en la situación prefuego es inferior a la unidad $(0,875)$, pero sí bastante elevado (Figura 10). Los valores caen bruscamente en el momento del fuego y se mantienen en valores muy bajos (inferiores a 0,4 ) hasta el final del período estudiado, indicando ausencia de recuperación vegetal respecto a las situaciones prefuego y control.

\subsubsection{Influencia de la situación previa al fuego en la dinámica posterior}

La tendencia mostrada por los cuatros grupos de puntos - establecidos en función de los umbrales ya citados del NDVI- es idéntica a la anteriormente comentada para el promedio de valores de las dos parcelas de Peñaflor (Figura 11). Las mayores diferencias se encuentran en el punto de partida de cada uno de estos grupos ya que el incendio tuvo un efecto homogeneizador llevando a todos ellos a valores de NDVI inferiores a 0,1 . Se observa en todo el período que los valores registrados por tres de los grupos $(1,2$ y 3$)$ tienen una correspondencia perfecta con la situación previa al fuego: el grupo 1 tiene los valores más bajos y los grupos 2 y 3 progresivamente más elevados. El grupo 4 (el de mayor NDVI prefuego), sin embargo, no reproduce esta tendencia y se sitúa por debajo de los grupos 2 y 3 durante la mayor parte del período de estudio.

De esta forma, y teniendo en cuenta que en la estación de Peñaflor no se ha producido regeneración vegetal, el NDVI prefuego influye en la dinámica posterior al fuego en los intervalos inferiores pero no en el de mayor nivel vegetal antes del fuego. Los puntos de este intervalo, precisamente por su mayor nivel vegetal, son los que experimentan mayores intensidades del fuego y los que sufren mayor daño en su estructura vegetal, dificultándose así su regeneración vegetal. En consecuencia, son los puntos con nivel vegetal intermedio antes del fuego $(0,4<\mathrm{NDVI}<0,6)$ los que mejor regeneran después del efecto distorsionador del fuego. 


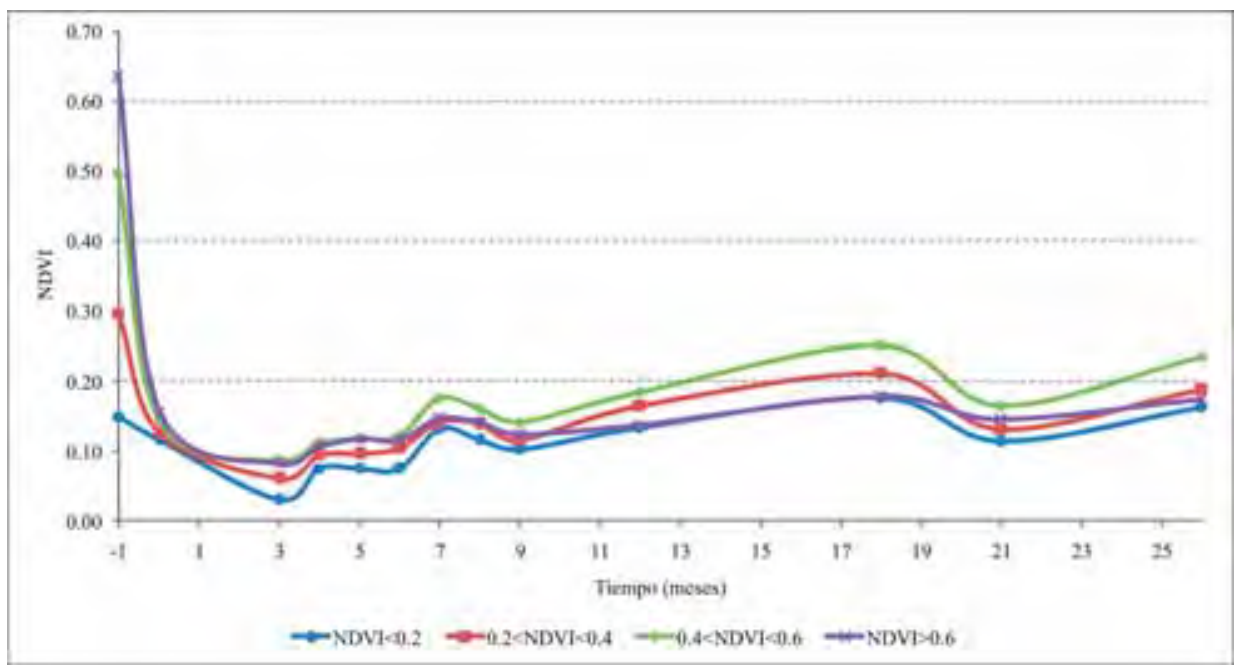

Figura 11. Evolución del NDVI para los cuatro grupos delimitados en función del NDVI prefuego en la estación de Peñaflor

\subsubsection{Influencia de la severidad en el proceso de regeneración vegetal}

Como ya se ha comentado anteriormente, el fuego en las parcelas de Peñaflor supuso la total destrucción de la cubierta vegetal. Fueron las áreas cubiertas de vegetación las que experimentaron un fuego intenso, mientras que las áreas de suelo desnudo circundantes no experimentaron en ellas el efecto directo del fuego. De esta forma, el estudio de la evolución de la severidad del incendio arroja diferentes resultados en función de que se tengan en cuenta o no los puntos de suelo desnudo muestreados.

Considerando los valores promedio de todos los puntos de las dos parcelas, la severidad del incendio medida con el índice dNDVI puede clasificarse como baja en el momento inmediatamente posterior al fuego $(\mathrm{dNDVI}=273)$. Los valores obtenidos en los meses posteriores al fuego son más elevados por la muerte de la vegetación parcialmente afectada por el fuego, durante estos meses los valores se sitúan en el intervalo de severidad moderada-baja (dNDVI? 300). Con la primera estación de crecimiento de la vegetación los valores vuelven a situarse en el intervalo de severidad baja permaneciendo allí hasta el final del período estudiado con variaciones correlativas al momento temporal del año (Figura 12).

Considerando sólo los puntos de vegetación, los valores de dNDVI se encuentran en todo el período en el intervalo de severidad moderada-baja. Los valores más elevados se registran en los meses posteriores al fuego, igual que ocurría en el análisis promedio de todos los puntos, y experimentan un progresivo descenso con variaciones que corresponden al transcurso de las estaciones del año, que generan pequeños cambios en la vegetación que modifican la estimación de la severidad (Figura 12). 


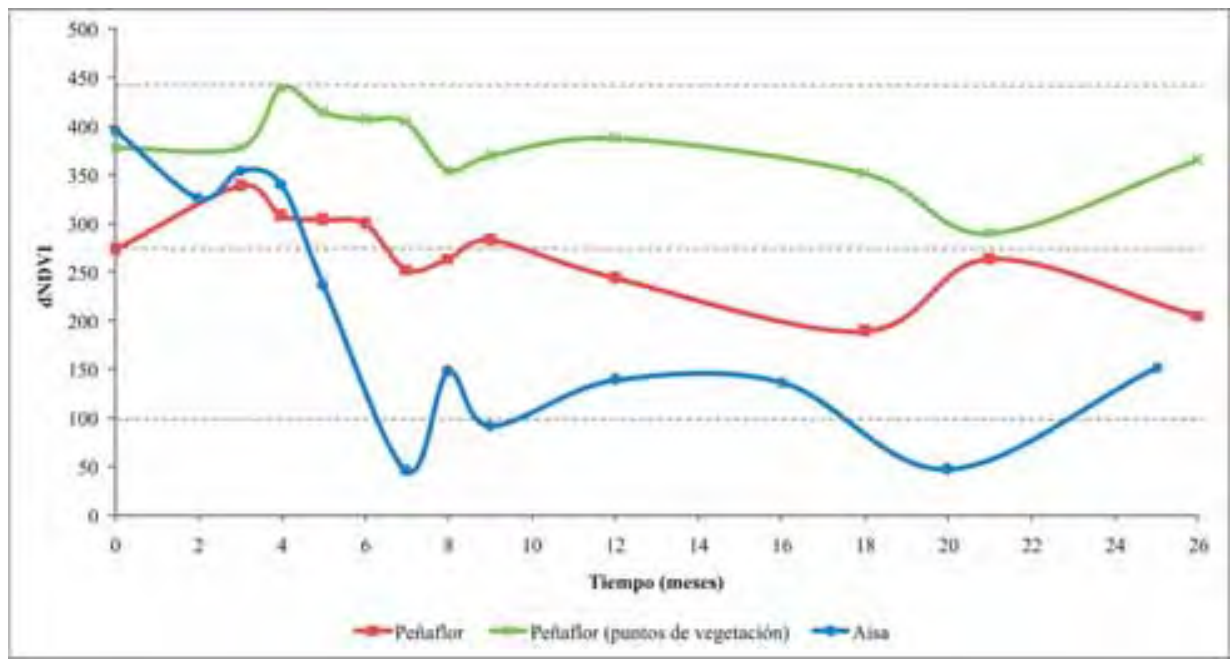

Figura 12. Evolución del índice de severidad dNDVI

El análisis de la severidad por grupos de NDVI prefuego muestra una clara diferenciación en los valores registrados por cada uno de estos grupos de puntos (Figura 13). El comportamiento observado es el mismo que el anteriormente comentado para el promedio de las parcelas pero cada uno de ellos se mantiene estable en un intervalo de severidad, indicando nuevamente la ausencia de regeneración vegetal en todos ellos y la estabilidad de su comportamiento postfuego.

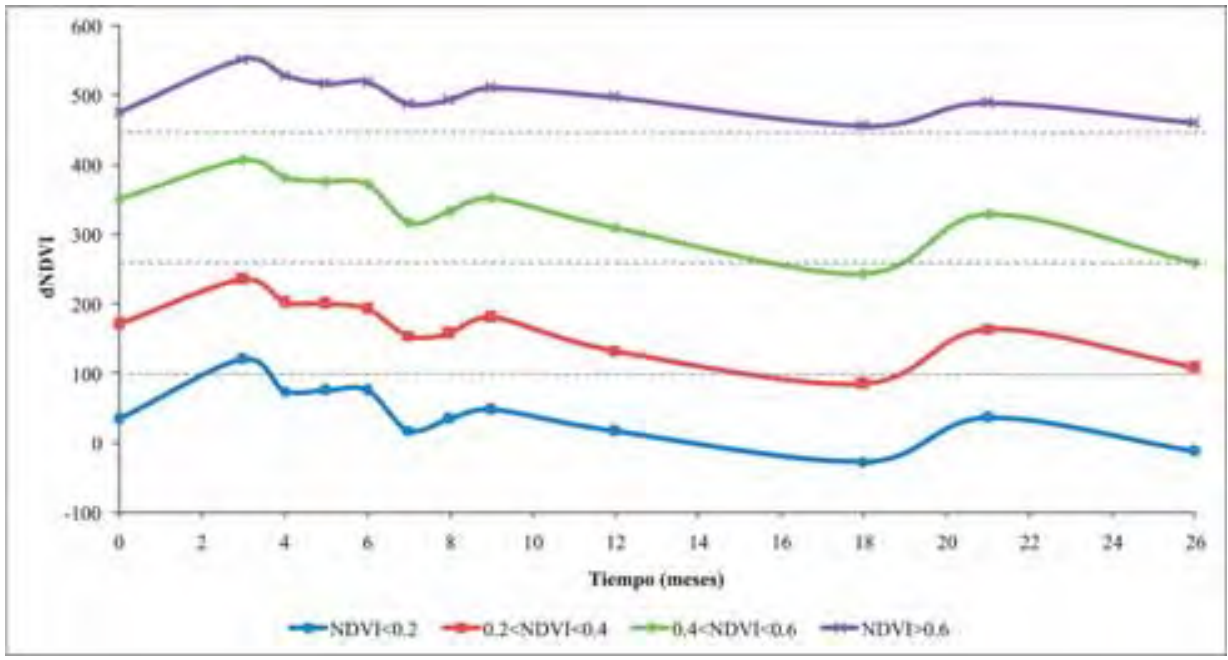

Figura 13. Evolución del dNDVI para los cuatro grupos delimitados en función del NDVI prefuego en la estación de Peñaflor 
Los puntos con NDVI prefuego inferior a 0,2 presentan en todo el período valores de severidad nula, hecho que corresponde con la realidad al tratarse de puntos no afectados directamente por el fuego. Los puntos con NDVI prefuego entre 0,2 y 0,4 se mantienen constantes en el intervalo de baja severidad, los puntos de nivel prefuego 0,4-0,6 se mantiene en el intervalo de severidad moderada-baja y, finalmente, el grupo de puntos con NDVI prefuego superior a 0,6 se sitúa siempre en el intervalo de severidad moderada-alta.

\subsection{Estación experimental de Aisa}

\subsubsection{Análisis de la regeneración vegetal postincendio}

Las dos parcelas que componen la estación experimental de Aisa evolucionan de forma idéntica en sus valores promedio individuales, lo que permite considerar sus valores promedio conjuntos como representativos de la dinámica general postincendio en un ambiente climático submediterráneo (Figura 14).

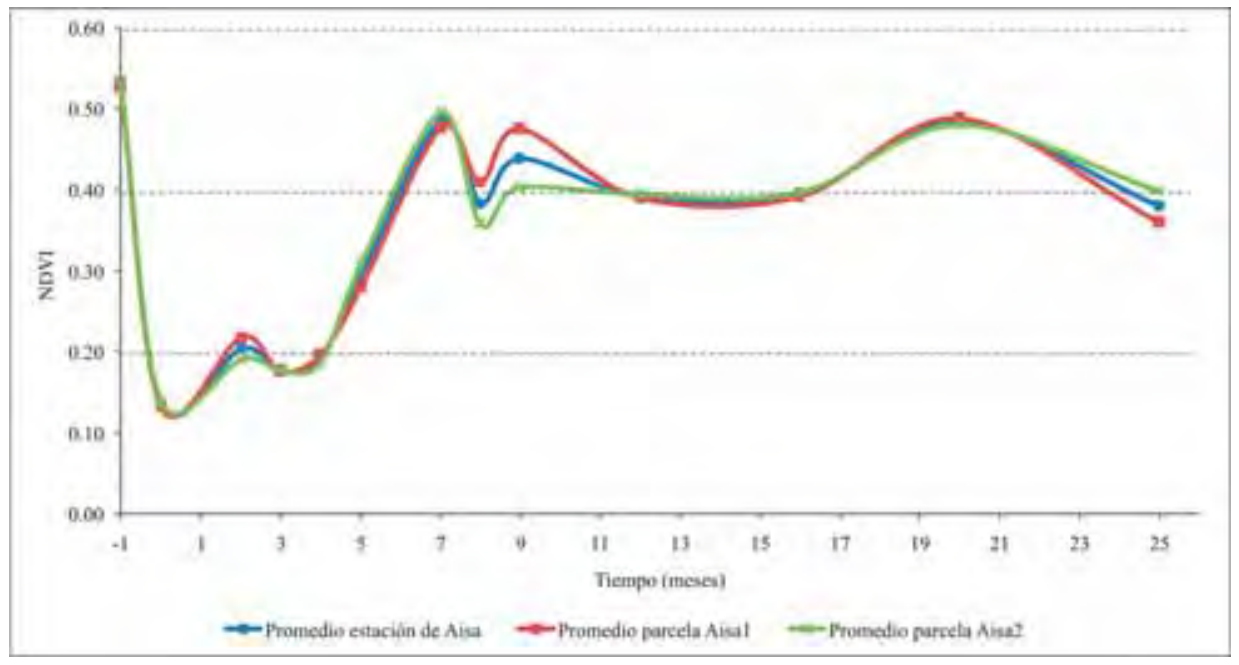

Figura 14. Evolución del NDVI en la superficie quemada de la estación experimental de Aisa

Las parcelas de Aisa parten de una situación previa al fuego de NDVI superior a 0,5 , considerado representativo de una vegetación activa y espacialmente densa (Figura 15). Este hecho corresponde con la realidad observable en las parcelas donde no pueden distinguirse áreas sin cubierta vegetal. El fuego provoca un descenso del nivel hasta valores inferiores a 0,15 , situación que puede considerarse como suelo desnudo y que deriva de la total desaparición de la vegetación (Figura 16). 


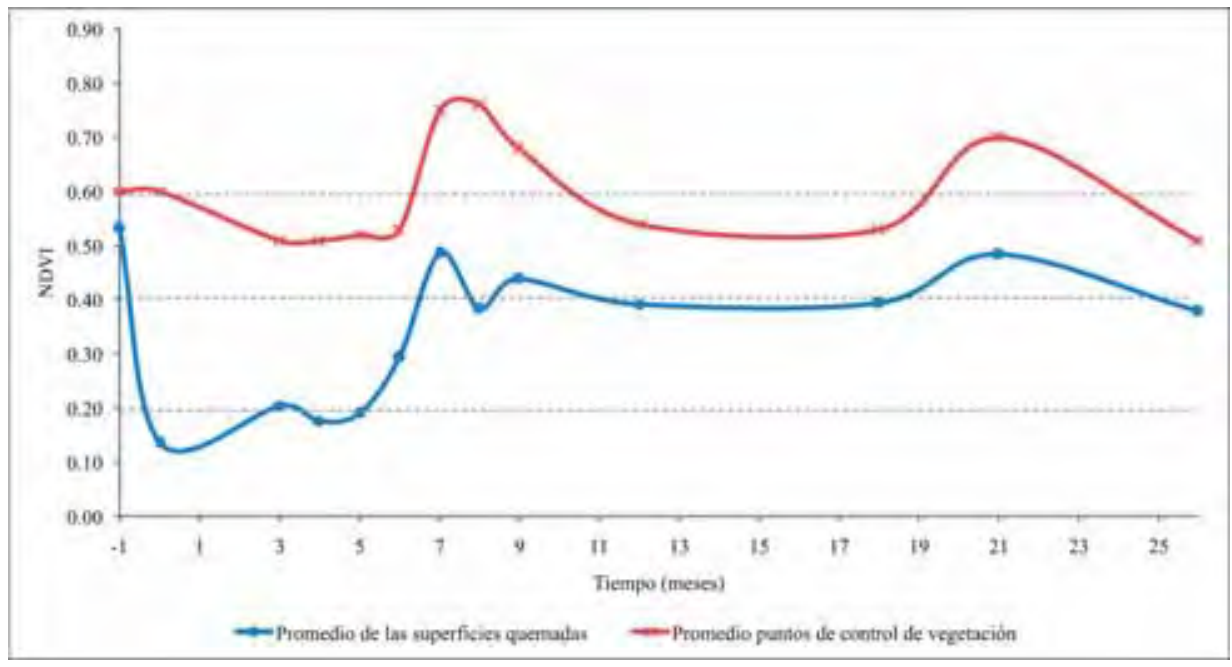

Figura 15. Evolución del NDVI en la estación experimental de Aisa
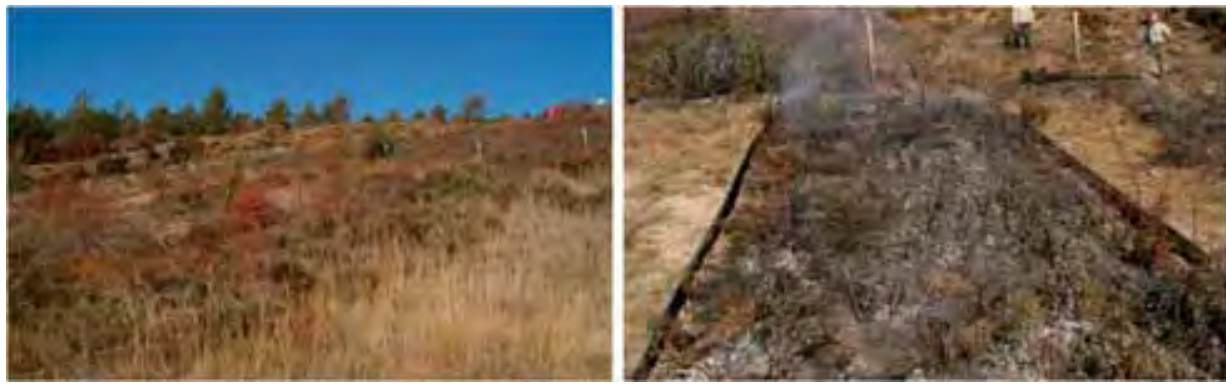

Figura 16. Fotografías de la estación de Aisa antes (izda.) y después del fuego (dcha.)

Desde el momento inmediatamente posterior al incendio las parcelas de esta estación empiezan a recuperarse, situándose por encima del umbral del suelo desnudo desde los dos meses posteriores al fuego y por encima del umbral de vegetación densa desde los seis meses, momento temporal que corresponde con la primera estación de crecimiento después del fuego. Desde este momento se mantiene por encima del umbral de vegetación, experimentando ciclos de ascenso y descenso asociados a las diferentes estaciones, ciclos que se reproducen exactamente en los puntos de control de vegetación.

\subsubsection{Evolución comparativa respecto a la situación control}

Las parcelas de la estación experimental de Aisa no presentan puntos de suelo desnudo, por ello no puede estudiarse la comparación de estos puntos con los puntos de control. Todos los puntos muestreados son de vegetación, y es de ellos de los que se estudia su evolución respecto a la situación control. 
Los valores de APC en el momento prefuego muestran una de alta correspondencia (valor de 0,9) entre los puntos de vegetación de las parcelas quemadas y los puntos de vegetación seleccionados para el control multitemporal (Figura 17). El momento inmediatamente posterior al fuego es cuando la distancia entre estos dos grupos de puntos es mayor (valor de 0,2), manteniéndose un comportamiento diferenciado hasta la primera estación de crecimiento, pero siempre con una tendencia general ascendente, es decir, de acercamiento entre el comportamiento de los puntos de vegetación quemados y el punto estable de control. El valor al final del período estudiado es de 0,75 , indicando una elevada recuperación respecto a la situación prefuego y una gran aproximación respecto a la situación control.

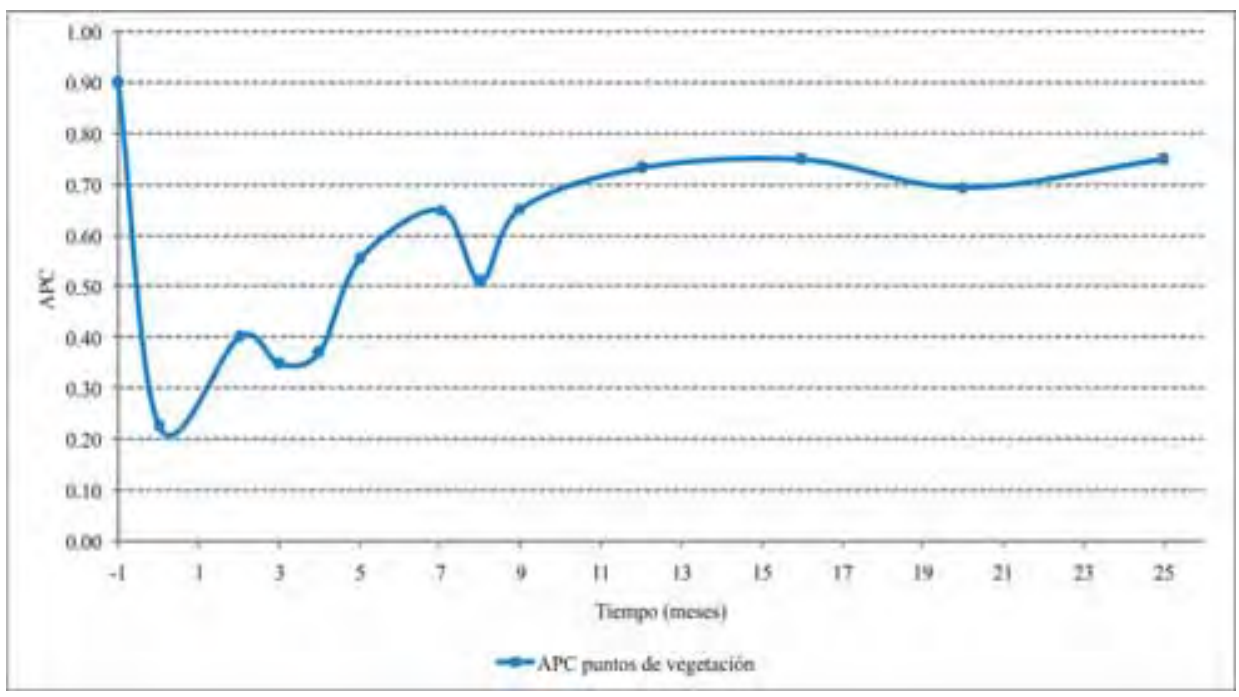

Figura 17. Evolución comparativa del APC en las parcelas de Aisa

El hecho de que no se alcance la situación de aproximación total con el prefuego o con el punto de control puede ser explicado por la diferente composición vegetal que se observa en las parcelas: dominio de las especies arbustivas en el prefuego y dominio de las especies herbáceas dos años después del fuego.

\subsubsection{Influencia de la situación previa al fuego en la dinámica posterior}

En las parcelas de Aisa, como ya se ha comentado anteriormente, sólo se observan puntos en el prefuego correspondientes a tres de los intervalos de clasificación del NDVI ( 2,3 y 4). La tendencia mostrada por estos tres grupos es igual a la comentada para el promedio de las dos parcelas (Figura 18). Los tres grupos muestran las mayores diferencias antes del fuego pero no así en el resto de la serie temporal estudiada, donde el 
grupo 2 se distingue claramente con valores de NDVI inferiores en 0,1 aproximadamente a los registrados por los grupos 3 y 4 que presentan valores prácticamente idénticos. De este modo, la influencia del NDVI prefuego en las parcelas de la estación de Aisa se reduce al intervalo de menor nivel $(0,2<\mathrm{NDVI}<0,4)$, no mostrando influencia en la regeneración vegetal de los grupos con NDVI superior a 0,4 .

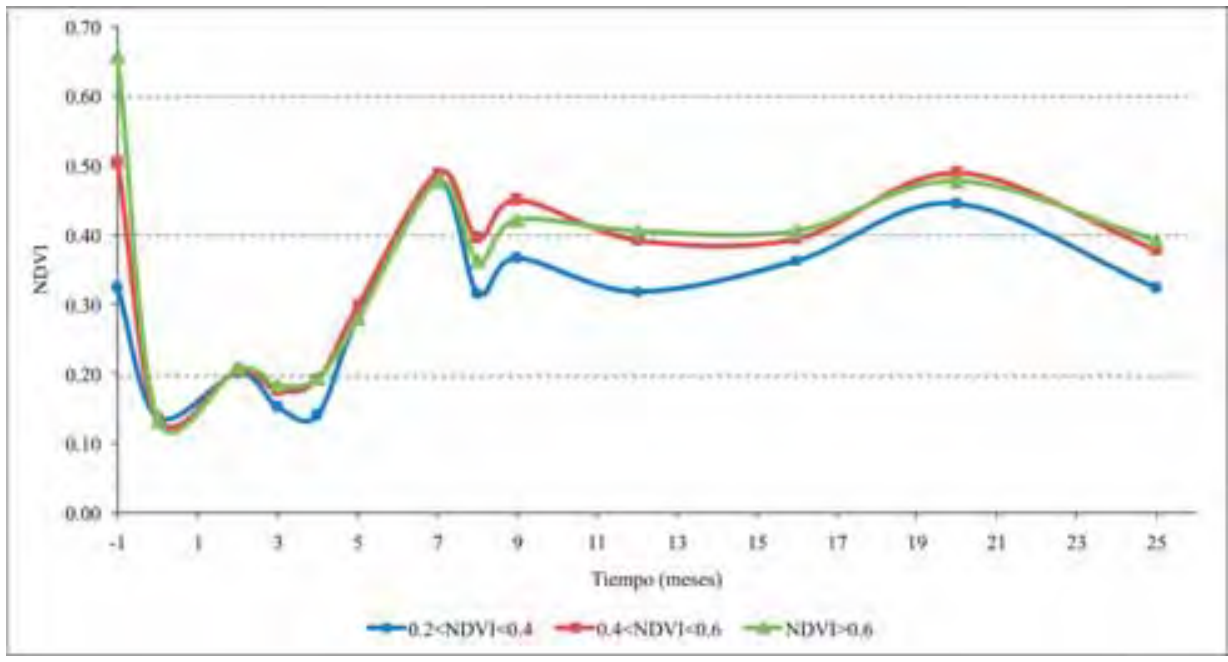

Figura 18. Evolución del NDVI para los tres grupos delimitados en función del NDVI prefuego en la estación de Aisa

\subsubsection{Influencia de la severidad en el proceso de regeneración vegetal}

La evaluación de la severidad en las parcelas de Aisa mediante el dNDVI estima una severidad inicial que se encuentra en el intervalo de severidad moderada-alta (Figura 12). Los valores de dNDVI permanecen en este nivel sólo en los tres meses posteriores al fuego ya que, con la llegada de la primera estación de crecimiento los valores, evolucionan hacia el intervalo de severidad baja e inmediatamente después hacia el intervalo de severidad nula o no quemado. A partir de este momento y durante el resto de la serie temporal oscila entre estos dos intervalos (severidad baja y nula) en función del estado de la vegetación, condicionado por la época del año.

El análisis de la severidad por grupos de NDVI prefuego muestra diferencias entre los tres grupos, aunque todos ellos mantienen una dinámica común que coincide con la anteriormente comentada para el promedio de las parcelas (Figura 19).

Los tres niveles de vegetación prefuego -0,2-0,4, 0,4-0,6 y superior a 0,6- presentan tres niveles distintos de severidad inmediatamente después del fuego -baja, media- 
baja y media-alta-, respectivamente. En los tres casos se produce una evolución paulatina hacia niveles de severidad inferiores, matizados por los cambios en la vegetación asociados a los diferentes períodos estacionales. De esta forma, los puntos de severidad inicial baja evolucionan hacia situaciones de severidad nula o, incluso, de revitalización vegetal respecto a la situación prefuego en algunas fechas. Los puntos de severidad inicial media-baja evolucionan hacia el intervalo de severidad nula en los meses de vigor vegetal o baja en los meses de baja actividad vegetal y, finalmente, los puntos de severidad inicial moderada-alta evolucionan hacia niveles de baja o moderada-baja en función de que la actividad vegetal sea alta o baja, respectivamente.

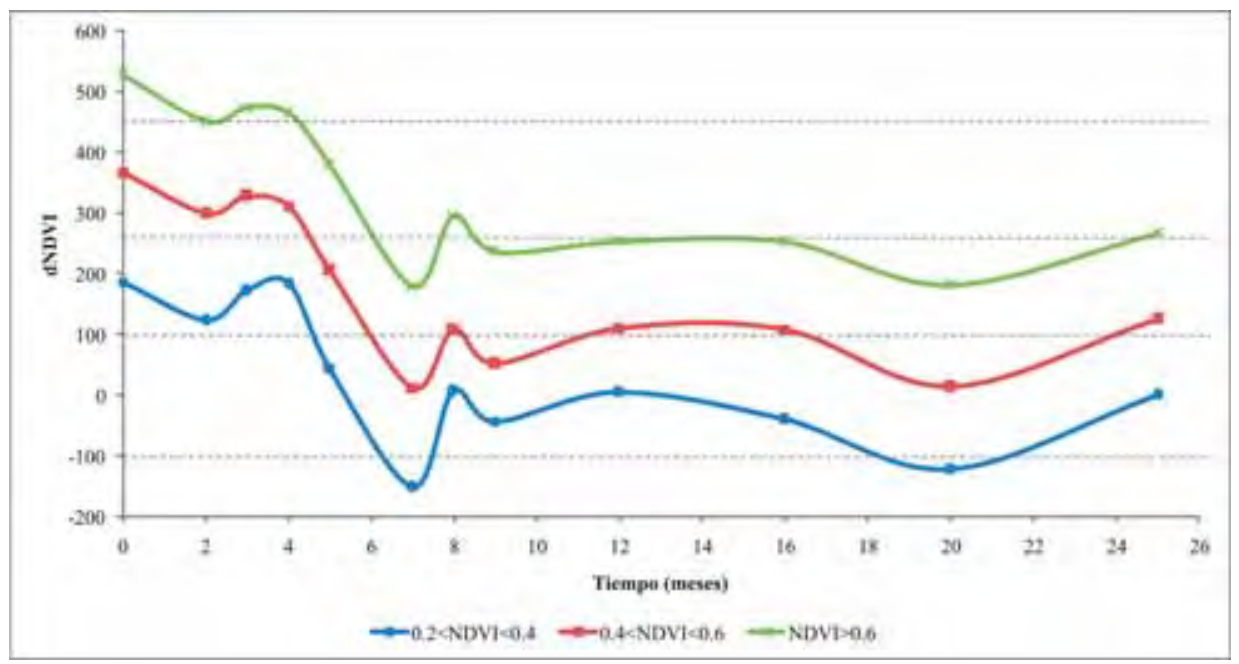

Figura 19. Evolución del dNDVI para los tres grupos delimitados en función del NDVI prefuego en la estación de Aisa

Cualquiera de las tres situaciones indica un proceso de recuperación vegetal y una matización de la severidad inicial con el transcurso del tiempo, especialmente con el paso de la primera estación de crecimiento después del fuego.

\subsection{Comparación del comportamiento postfuego entre estaciones experimentales}

El estudio individual de las dos estaciones experimentales permite observar importantes diferencias entre ellas (Figura 20). Estas diferencias existen ya en la situación anterior al fuego, no tanto en la composición vegetal de las parcelas cuanto en su estructura espacial, con vegetación densa y ausencia de áreas de suelo desnudo en la estación de Aisa y distribución en parches de vegetación rodeados de suelo desnudo en la estación de Peñaflor. 
Esta diferente estructura espacial tuvo su repercusión inmediata en las características del fuego, tal como se ha comentado anteriormente, alcanzando mayor intensidad y severidad a corto plazo en la estación de Aisa respecto a la de Peñaflor.



Figura 20. Comparación de la evolución del NDVI en las superficies quemadas de las dos estaciones experimentales

Después del fuego, las diferencias en el comportamiento de ambas estaciones son, incluso, más acusadas. Partiendo de una idéntica situación postfuego con NDVI ? 0,15, la estación de Aisa alcanza niveles de NDVI superiores al umbral de vegetación siete meses después del fuego, con la llegada de la primera estación de crecimiento en los meses de primavera. Mientras tanto, la estación de Peñaflor continúa todo el período de estudio por debajo del umbral de suelo desnudo, a pesar de que se observan leves incrementos en los momentos correspondientes a los meses primaverales. De este modo, mientras que en Aisa el proceso de regeneración vegetal en términos de recubrimiento superficial se completa en apenas siete meses, en Peñaflor todavía no ha tenido lugar transcurridos dos años desde el fuego. Aunque el proceso de regeneración vegetal en Aisa se considere completado desde el punto de vista superficial en la primera primavera tras el fuego, es necesario indicar que no puede considerarse finalizado desde el punto de vista de la composición vegetal. Antes del fuego las parcelas están compuestas por vegetación arbustiva, pero tras la regeneración inicial es la vegetación herbácea la dominante; desde este momento hasta el final del estudio se ha observado en las parcelas una progresiva sustitución de esta vegetación herbácea por la original de porte arbustivo, aunque no se ha alcanzado la situación original.

El estudio del comportamiento por grupos de NDVI prefuego ofrece diferencias menos acusadas entre estaciones. Considerando que ninguno de los intervalos ha rege- 
nerado en la estación de Peñaflor, los intervalos de nivel de vegetación intermedio en el prefuego parecen ser los que muestran mayor capacidad al presentar valores ligeramente más elevados. En Aisa, donde todos los intervalos han experimentado regeneración, las diferencias se observan entre los grupos por encima y por debajo del umbral 0,4.

La estimación global de severidad inmediata considera ambas estaciones con un nivel de severidad moderado-bajo, en ambos casos con diferencias internas en función del nivel de vegetación prefuego, tal como se ha explicado anteriormente en el análisis individual de ambas estaciones. Las diferencias se observan en la evolución posterior y son explicadas por la presencia o ausencia de procesos de regeneración vegetal en Aisa y Peñaflor, respectivamente. Su presencia en Aisa hace disminuir progresivamente el nivel de severidad hacia severidad nula o incluso revitalización vegetal en algunos intervalos de NDVI previo y su ausencia en Peñaflor mantiene los niveles de severidad constantes en todo el período.

\section{Conclusiones}

Este trabajo muestra la validez del empleo de técnicas de radiometría de campo para el estudio de la regeneración vegetal postincendio en el contexto metodológico de las parcelas experimentales. Por una parte, este marco espacial permite un control total de la realidad estudiada y de los procesos que tienen lugar en ella, asegurando al mismo tiempo la total correspondencia entre medidas, aspecto fundamental en un estudio multitemporal como el aquí mostrado. Por otra parte, la información espectral obtenida mediante un radiómetro de campo ofrece información objetiva sobre el estado de la vegetación en las parcelas estudiadas y permite establecer una equivalencia con la visión que de esas mismas parcelas obtendría un sensor remoto, abriendo así la posibilidad a la aplicación de índices generales de teledetección y a una futura aplicación a escala regional de los resultados obtenidos en esta escala de detalle.

El registro continuado de la información durante dos años y la comparación de dos estaciones experimentales sólo diferenciadas por su ambiente climático han permitido obtener importantes conclusiones sobre la dinámica vegetal posterior al fuego y sobre la importancia de las diferentes variables con influencia en esta dinámica.

De forma general, la información analizada permite establecer la siguiente conclusión: la estación experimental de Aisa, de ambiente submediterráneo, presenta una gran capacidad de regeneración vegetal postfuego; por el contrario, la estación experimental de Peñaflor, de ambiente semiárido, no muestra en el mismo período capacidad de regeneración vegetal. Los procesos de regeneración vegetal en Aisa se han producido de forma rápida y eficaz en términos de cubrimiento superficial, conduciendo a una total recuperación con la llegada de la primera estación de crecimiento de la vegetación. La eficacia no ha sido igual en términos de composición vegetal, apreciándose un cambio hacia especies herbáceas y una progresiva evolución hacia las originales especies arbustivas hasta el final del período estudiado. En la estación de Peñaflor los procesos de regeneración vegetal no han tenido lugar de forma generalizada en los dos años analizados, siendo observable la 
vegetación sólo en muy puntuales localizaciones que la mantuvieron después del fuego. Los valores generales mostrados en todo el período son los típicos de áreas de suelo desnudo. En este sentido, se puede considerar que el fuego ha tenido un efecto mucho más perjudicial en el ambiente semiárido, ya que los efectos negativos derivados de la desaparición de la cubierta vegetal (incremento de las tasas de erosión y pérdida de suelo) se han prolongado durante un período de tiempo muy superior.

La variable empleada para estudiar la regeneración vegetal, el índice de vegetación NDVI, ha demostrado su validez como estimador global del recubrimiento vegetal y su evolución temporal en las parcelas. No obstante, ha mostrado algunas limitaciones en relación con la sensibilidad a la composición vegetal. En este sentido, las técnicas hiperespectrales de tratamiento de la información radiométrica ofrecen un amplio abanico de posibilidades. Análisis basados en la extracción de rasgos de absorción o en el cálculo de primera derivada de la información espectral presentan buenas perspectivas de cara no sólo a mejorar la cuantificación del nivel vegetal sino a estimar determinados parámetros que permitan la precisa identificación de la composición vegetal y su estado (Zarco et al., 2001).

En líneas generales, la evolución temporal del NDVI ha mostrado poca sensibilidad a las variables explicativas estudiadas. En consecuencia, puede considerarse que en este tipo de comunidades vegetales la variable climática es la que realmente condiciona los procesos de regeneración vegetal.

La variable nivel de NDVI previo tiene una influencia muy baja en ambas estaciones experimentales: (1) en Aisa sólo se observan diferencias entre los grupos con NDVI prefuego superior o inferior a 0,4, el valor establecido como umbral de vegetación; (2) en Peñaflor, a pesar de la ausencia general de regeneración, las áreas con NDVI prefuego intermedio $(0,2-0,6)$ muestran mayor capacidad que las áreas de mayor NDVI prefuego (superior a 0,6 ).

Del mismo modo, la variable severidad estimada con el índice dNDVI inmediatamente después del incendio tampoco ejerce influencia en la evolución de la vegetación. El valor de dNDVI inicial se encuentra totalmente determinado en ambos casos por el NDVI previo al fuego. Desde esta situación inicial las dos estaciones evolucionan de acuerdo a la tendencia comentada: (1) en AISA, todos los niveles de severidad muestran evolución hacia intervalos de severidad inferiores, finalizando el período en niveles de severidad baja o nula; (2) en Peñaflor, los valores de severidad inicial no varían al no producirse regeneración vegetal.

El estudio evolutivo de los niveles de severidad ha permitido verificar en estos ambientes las teorías generales existentes en la bibliografía sobre la necesidad de retrasar la evaluación de la severidad después de la primera estación de crecimiento de la vegetación (Key y Benson, 2004). Considerando una perspectiva a corto plazo, ambos ambientes muestran el mismo nivel de severidad; sin embargo, considerando una perspectiva a medio plazo y dando tiempo a la vegetación a mostrar su capacidad de res- 
puesta, la severidad ha sido muy superior en el ambiente semiárido, que no ha sido capaz de regenerarse.

Por otra parte, la escasa influencia mostrada por la variable dNDVI invita a la realización de estudios que mejoren la estimación de la variable severidad ampliando la información radiométrica a nuevas regiones del espectro como el infrarrojo medio, aplicando técnicas hiperespectrales de tratamiento de la información radiométrica (Parra, 2006), o elaborando nuevos índices con longitudes de onda especialmente sensibles a los productos superficiales resultado de la combustión de la vegetación. Con este objetivo, la información aportada por fotografías verticales de alta resolución espacial sería de gran utilidad, al permitir una exacta cuantificación de la cantidad de combustible consumido por el fuego y de los productos superficiales resultado de esta combustión (Montorio et al., 2006).

Del mismo modo, en el marco metodológico de las parcelas experimentales, el monitoreo de los procesos superficiales mediante esta técnica de fotografía de alta resolución aportaría información muy valiosa sobre la dinámica postfuego (regeneración vegetal y procesos erosivos), permitiendo su exacta espacialización, como complemento de la información radiométrica y mejora de tradicionales técnicas de trabajo de campo (Pérez-Cabello et al., 2006).

\section{Agradecimientos}

Este estudio fue financiado por una beca provista por el Gobierno de Aragón y contó con el soporte del Ministerio de Ciencia y Tecnología español y del Gobierno de Aragón, a través de los proyectos RS-FIRE (CGL2005-04863) y PIR-FIRE (PIP098/2005), respectivamente.

\section{Referencias bibliográficas}

Díaz-Delgado, R. y Pons, X. (2001). Spatial patterns of forest fires in Catalonia (NE of Spain) along the period 1975-1995. Analysis of vegetation recovery after fire. Forest Ecology and Management 147: 67-74.

HANES, T.J. (1971). Succession after fire in the chaparral of southern California. Ecol. Monogr. 41: 27-52.

Key, C.H., y Benson, N.C. (2004). Landscape Assessment (LA) Sampling and Analysis Methods. Disponible en <http://www.fire.org./firemon/lc.html>

MAY, T. (1991). Observaciones y reflexiones sobre el comportamiento tras fuego de algunas especies de la zona mediterráneas de Andalucía Oriental. Ecología 5: 125-134.

Montorio, R., Pérez-Cabello, F., García-Martín, A., Palacios, V., De la Riva, J., Echeverría, M.T., Ibarra, P. y Lasanta, T. (2006). Methodology approach for 
analyzing relationships between fire severity levels and reflectance values. Forest Ecology and Management 234S: S108.

MilleR, J.D. y Yool, S.R. (2002). Mapping forest post-fire canopy consumption in several overstory types using multi-temporal Landsat TM and ETM+ data. Remote Sensing of Environment 82: 481-496.

NAVEH, Z. (1990). Fire in the Mediterranean-A landscape ecological perspective. En Fire in Ecosystem Dynamics (Goldammer, J.G. y Jenkins, M.J. Eds.). SPB Academic Publ., 1-20.

PARra, A. (2005). Estimación de niveles de severidad del fuego a partir de datos hiperespectrales EO-1 HYPERION. Tesis Doctoral. Departamento de Geografía, Universidad de Alcalá, Alcalá de Henares.

PAtTerson, M.W. y Yool, S.R. (1998). Mapping fire-induced vegetation mortality using Landsat Thematic Mapper data: a comparison of linear transformation techniques. Remote Sensing of Environment 65: 132-142.

Pérez-Cabello, F., Montorio, R., García-Martín, A., Palacios, V., De la Riva, J., ECHEVERríA, M.T., IBARRA, P. y LASANTA, T., 2006. Monitoring post-fire processes in semiarid and sub-Mediterranean environments using high spatial resolution photograph. Forest Ecology and Management 234S: S182.

Rouse, J.W., HaAs, R.H., Schell, J.A., Deerino, D.W. y Harland, J.C. (1974). Monitoring the vernal advancement of retrogradation of natural vegetation. NASA/OSFC. Type III. Final Report. Oreenbello MD, pp. 371.

Schaepman, M., Koetz, B., Schaepman-Strub, G. y Itten, K. (2005). Spectrodirectional remote sensing for the improved estimation of biophysical and chemical variables: two case studies. International Journal of Applied Earth Observation and Geoinformation 6: 271-282.

Van Wagtendonk, J., Root, R. y Key, C.H. (2004). Comparison of AVIRIS and Landsat ETM+ capabilities for burn severity. Remote Sensing of Environment 92: 397-408.

Zarco-Tejada, P.J., Miller, J.R., Nolands, T.L., Mohammed, G.H. y Sampson, P.H. (2001). Scaling-Up and Model Inversion Methods with Narrowband Optical Indices for Chlorophyll Content Estimation in Closed Forest Canopies with Hyperspectral Data. IEEE Transactions on Geoscience and Remote Sensing 39 (7): 1491-1507. 